\title{
Agent-Based Simulation of Customer Satisfaction : A Systematic Review
}

\author{
Ruth Appiah ${ }^{*}$, Lulin Zhou ${ }^{1}$, Emmanuel Bosompem Boadi ${ }^{2}$, Bentil Anthony Ewusi ${ }^{1}$, Andrews Minkah ${ }^{1}$, \\ Abigail Larnyo ${ }^{1}$ \\ ${ }^{1}$ School of Management, Jiangsu University, Zhenjiang 212013, P.R. China. \\ ${ }^{2}$ School of Public Administration, Hohai University, Nanjing 202001, P.R. China. \\ Corresponding Author: *ruthapp27@yahoo.com
}

\begin{abstract}
Article Info

Volume 8, Issue 5

Page Number : 278-290

Publication Issue

September-October-2021

Article History

Accepted : 01 Oct 2021

Published : 07 Oct 2021

Mathematical modelling of customer satisfaction has attracted strong academic interest over the centuries. Traditional satisfaction models have aimed at empirical generalizations and hence describe the customers' behaviour parsimoniously at the market level. More recently, agent-based modelling and simulation has increasingly been adopted since it operates on the individual level and, thus, can capture complex emergent phenomena highly relevant in satisfaction research. Agent-based methods have been applied in this context both as intuition aids that facilitate theory-building and as tools to analyse realworld scenarios, support management decisions and obtain policy recommendations. This review addresses both streams of research. The research critically examine the strengths and limitations of agent-based modelling in the context of customer satisfaction. The target audience of the paper includes both researchers in marketing interested in new findings from the agent-based modelling literature and researchers who intend to implement agent-based models for their own research endeavours. Accordingly, cover pivotal modelling aspects in depth (concerning, consumer behavior) and outline existing models in sufficient detail to provide a proper entry point for researchers new to the field.

Keywords : Agent-based modelling, Simulation, Customer satisfaction
\end{abstract}

\section{INTRODUCTION}

Mathematical modelling of customer satisfaction has attracted strong academic interest since a number of pioneering works by Maharjan \& Khadka.Kabu (2017). In particular, the model modernized by $\mathrm{Ng} \& \mathrm{Luk}$, (2019), which characterizes the satisfaction of an innovation as a contagious process that is initiated by 
mass communication and propelled by word-ofmouth, is widely cited and was selected as one of 5 most influential papers in the past decades of Management Science (Hopp 2016) as in (Wang \& Yu, 2017).

Management practitioners also show considerable interest in satisfaction models because firms' ability to successfully satisfy customers determines whether they can create competitive advantage and secure long-term success. Managers can therefore benefit considerably from tools that help them to preestimate the market response to new products, provide model-based decision-support, and allow them to assess new product introduction strategies.

Aggregate models such as the Bass model, which provides an empirical generalization based on a differential equation formulation, provide such support only to a limited degree, as they are not designed for what-if type questions. Furthermore, these models do not explicitly consider consumers' heterogeneity and the complex dynamics of social processes that shape the satisfaction and can therefore tackle only a limited set of theoretical issues. Aggregate models have also been criticized for a lack of predictive and explanatory power.

To overcome these limitations and open up new research opportunities, agent-based modelling and simulation has increasingly been adopted in satisfaction research in recent years. This trend is in line with a broader development in the social sciences (the overview provided by Squazzoni (2010) as in (Priporas et al., 2017). One reason why the use of this bottom-up methodology has gained momentum in recent years lies in its ability to model complex emergent phenomena such as the satisfaction of an innovation in a socio-economic system that more traditional modelling approaches cannot capture easily. In agent-based satisfaction models, the atomic model element is not the social system as a whole, but the individual consumer or agent. Consumers' heterogeneity, their social interactions and their decision making processes can be modelled explicitly.
The macro-level dynamics of the social system emerge dynamically from the aggregated individual behavior and the interactions between agents.

The literature on agent-based models (hereafter ABMs) of customer satisfaction can be divided into two major streams. The first stream is aimed at theoretical insights and is therefore concerned with highly abstract and generic representations of satisfaction processes. The second stream, which has experienced significant growth in recent years, is concerned with the practical application of ABMs to provide forecasts, decision support, and policy analyses for specific applications based on empirical data.

The current paper addresses both streams of research. While customer satisfaction models and their applications have been reviewed extensively over the past decades (Mahajan and Muller 2000; Mahajan et al. 1990; Sultan et al. 2002; Parker 2004; Mahajan et al. 1995, 2002; Meade and Islam 2006) as in (Rudolfné Katona \& Komáromi, 2016), far less attention has been paid to the fast growing literature on agentbased satisfaction models. A number of reviews have addressed related aspects: Garcia (2015) outlines (potential) uses of ABMs in innovation/new product development research, Dawid (2016) reviews agentbased computational economics models of innovation and technological change, Peres et al. (2018) broadly review satisfaction modelling efforts, and Hauser et al. (2016) summarize research on innovation from a marketing perspective. The literature on ABMs of customer satisfaction, however, has not been reviewed so far. Thus, this study seeks to address the gap in literature by providing an overview on ABMs' impact in customer satisfaction by (i) discussing key modelling aspects; (ii) evaluating theoretical advances that ABMs have contributed to satisfaction research (iii) reviewing $\mathrm{ABMs}$ ' practical impact in terms of applications to real-world problems.

\section{SYSTEMATIC LITERATURE REVIEW}

A systematic literature review was conducted to assess the progress of research on the use of agent based simulation in customer satisfaction, by so doing 
identify the knowledge gap in the area of the research. The Preferred Reporting Items for Systematic Reviews and Meta-Analyses (PRISMA) framework was used to perform the literature review.

The PRISMA framework is an evidence-based minimum set of items aimed at helping authors to report a wide array of systematic reviews and metaanalyses that assess the benefits and harms of agent based Simulation.

A search was conducted for existing literature on on the use of agent based simulation in customer satisfaction. In order to remove any biases from the systematic review process, the following inclusion and exclusion criteria were set out. For a literature to meet the inclusion criteria, it must first of all be either a research journal, original article, review article, encyclopedia, book chapter, conference abstracts, conference article, correspondence, discussion, editorial, mini review, and or practice guideline. Secondly, such articles must be published in the English Language. Finally, articles have to either be a primary or secondary outcome evaluations on usability and user experience, adoption barriers and enhancers, design and user's level of satisfaction with the customers. Articles on customer satisfaction published before 2016 were excluded from the study. Literature search was conducted in online databases such as Emerald Insight, ScienceDirect and Google Scholar.

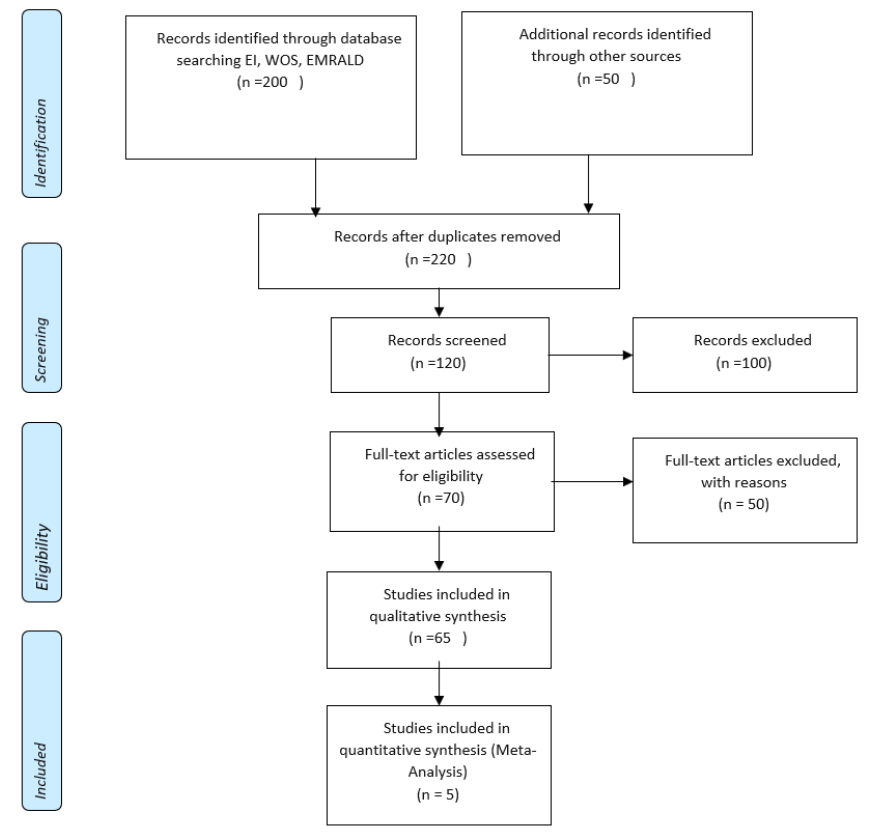

Figure 1 above shows the flow diagram of the PRISMA systematic review steps. The literature search returned 250 articles published from 2016 to 2020 in various academic journals. Of the total search, 30 were removed due to article duplication, leaving a total of 220 articles. 100 unrelated articles were also deleted in order to provide a very concise and content-relevant articles for analysis. 120 articles were screened to determine if they were relevant to the objectives set out for this literature review. After the screening, 50 articles were removed because they did not meet the inclusion criteria outlined above. Of the 70 titles, abstracts and full-text articles, assessed for eligibility, 65 were further deleted based on the inclusion criteria. The remaining 5 articles were critically synthesized for meta-analysis and discussed.

\section{RESULTS}

\section{Agent-based modelling of customer satisfaction}

In this section, the aim is to discuss different strategies for modelling consumer behavior and social influence, two key aspects in agent-based models of innovation satisfaction. 


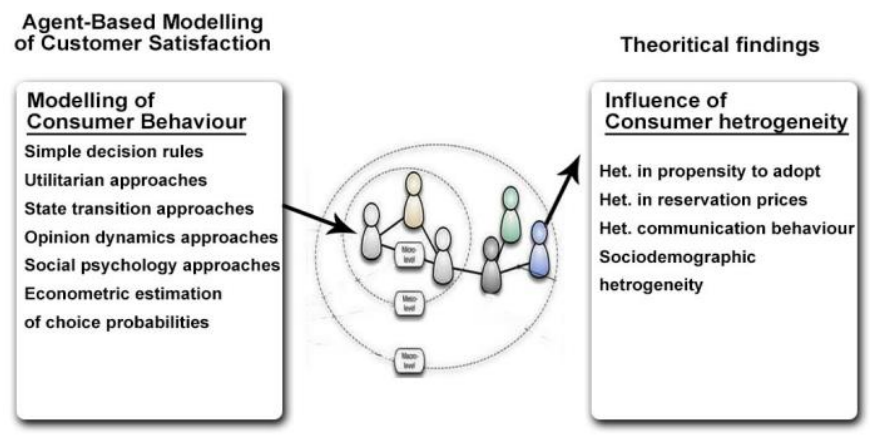

Fig. 2 Key elements of an agent-based satisfaction model and theoretical findings of this review

To this end, the research systematically identify different approaches and point to specific models in the literature as illustrations. Figure 1 illustrates key elements of an agent-based satisfaction model (consumer agents and their interactions).

\section{Modelling of consumer behaviour}

A pivotal element of agent-based satisfaction models is the explicit representation of consumers' decision making processes, most importantly those related to the decision to adopt an innovation (or to reject it, which, however, is not considered explicitly in most models). A number of both deterministic and stochastic approaches have been developed to model these decisions, ranging from simple decision rules to sophisticated psychological models. In the following, the most common approaches were examined (Khazaii, 2016; Tong et al., 2018).

\section{Simple decision rules}

Perhaps the simplest conceivable decision rule is to adopt as soon as the first of an agent's acquaintances has adopted. This rule can be interpreted as a contagious spread of information about the innovation. Threshold models use similar mechanisms, but typically stipulate that a consumer adopts only once a certain proportion of its acquaintances has adopted. The threshold is typically varied across the population and either deterministic, i.e., agents decide deterministically once the threshold is reached (e.g., Alkemade and Castaldi 2015), or probabilistic, i.e., agents adopt with a certain probability once the threshold is reached (e.g., Bohlmann et al. 2017).

Satisfaction models in the economics literature (e.g., Kocsis and Kun 2018; Hohnisch et al. 2018; Cantono and Silverberg 2019; Faber et al. 2016) typically use simple decision rules based on cost minimization or heterogeneous reservation prices. These models frequently assume falling prices due to learning effects and tend to interpret social influence as benefits due to network externalities. These network externalities occur when the utility of a network good increases with the number of peers or the share of the market that has adopted (Katz and Shapiro 1986, 1992) as in (Nyadzayo \& Khajehzadeh, 2016).

\section{Utilitarian approaches}

From a classical rational choice perspective, customer satisfaction phenomena pose an explanatory challenge. They do not fit directly into classical economic thinking because homogeneous, perfectly rational individuals acting in a perfect market with complete information would always adopt at the same time. When we acknowledge that individuals are neither homogeneous, nor perfectly informed, (expected) utility is an obvious candidate concept for modelling adoption decisions, given that it constitutes a key building block of standard microeconomic theory of individual choice behaviour. One could therefore expect utility theoretic approaches to feature prevalently in the literature. Surprisingly, however, the number of contributions that analyse customer satisfaction in a utilitarian framework is limited. Many of them use "utility" as an interpretive tag rather than explicitly modelling the choice between a single or multiple innovations and non-adoption (i.e., utility of highest alternative opportunity) by means of utility functions that represent individual preferences. Delre et al., (2017), for example, formulate threshold functions for individual utility based on 
heterogeneous "quality expectations" and social utility components to obtain a utility aspiration level for each consumer agent. Conceptually, their approach does not differ fundamentally from other threshold models, apart from the interpretation of thresholds as "utility aspiration levels". In a similar vein, Choi et al. (2018) introduce a fixed individual utility component which is interpreted as a "quality perception" and formulate social utility, which they interpret as benefits due to network externalities, as a linear function of the proportion of adopters in the neighborhood.

Few attempts have been made to integrate multiattribute preference modelling approaches (for an introduction to multi-criteria decision making, Keeney and Raiffa 2017) into ABMs of customer satisfaction so far.

\section{State transition approaches}

A number of models represent adoption behavior by means of a single dichotomous variable that represents agents' external state, i.e., agents are either in a "potential adopter" or an "adopter" state. In this respect, state-transition-based customer satisfaction models differ from many infectious disease models, which are frequently referred to as an inspiration and analogy for customer satisfaction models, since these models typically use more than two states (e.g., SEIR - susceptible, exposed, infected, removed / recovered). Goldenberg and Efroni (2017), for example, model adoption as a probabilistic transition between two states that results either from spontaneous transformation or from WoM induced awareness.

Other models, by contrast, represent the decision making process as a sequence of transitions between more than two states. Goldenberg et al. (2017), for example, consider rejection explicitly and specify separate transition probabilities for adoption/rejection based on positive WoM, advertising, and negative WoM. Deffuant et al. (2015), use a fixed state transition scheme based on interest (no, maybe, yes) and information states (not-concerned, information request, no adoption, pre-adoption, adoption). Thiriot and Kant (2018) also model adoption decisions as a sequence of transitions between multiple states, viz. awareness, information seeking, adopter, WoM spreading.

\section{Opinion dynamics approaches}

Opinion dynamics in social systems have been studied intensively in recent years (Kocsis and Kun 2008). For an introductory article, this paper refers to Hegselmann and Krause (2017). A number of customer satisfaction models have adopted ideas from the rich stream of opinion dynamics literature, stipulating that consumers develop preferences in a collective process of opinion formation. In a so-called CODA (continuous opinions, discrete actions) model put forward by Martins et al. (2019), for example, each agent has a probabilistic opinion assigned to the proposition " $\mathrm{A}$ is the best choice that can be made". This opinion is updated by means of Bayesian interference based on observed adoption behavior of neighboring agents. Refusal in adopting is increasingly weighted by neighbor agents as evidence against the innovation. Deroïan (2016) simulates the emergence of a collective evaluation of an innovation based on individual propensities to adopt that are interpreted as opinions. The author incorporates the idea of "bounded confidence" (Hegselmann and Krause 2018) by assuming that consumers with similar opinions tend to form stronger bonds while those with very different opinions tend to diminish the level of received influence.

\section{Social psychology approaches}

Social psychology approaches, arguably the most sophisticated and least parsimonious, are based on psychological theories of behavior. Rather than representing consumers as instances of homo 
economicus, these models incorporate the behavioural richness exhibited by "homo psychologicus" in real life (Jager et al. 2019). Adoption decisions are therefore based on psychological rules rather than perfect rationality. For a comparison of the suitability of various social psychological theories for consumer agent design, we refer to Zhang and Nuttall (2019).

Ajzen's theory of planned behavior (TPB, Ajzen 2017) is a commonly used theoretical foundation for modelling consumer agents' behavior in application and policy-oriented satisfaction models. It postulates that attitude, perceived behavioral control, and intention are predictors of behavior. Kaufmann et al. (2019) use TPB to model the satisfaction of organic farming practices. Agents (i.e., farmers) adopt if their intention exceeds an empirically derived threshold. Schwarz and Ernst (2019) use TPB as a framework to model consumers' decisions to adopt or reject watersaving innovations using two different kinds of decision rules: a cognitively demanding deliberate decision rule and a very simple decision heuristic. Zhang and Nuttall (2018) model smart metering adoption behavior based on TPB.

\section{Econometric estimation of choice probabilities}

While theoretical models need to be less concerned with methods for initializing the simulation with empirical data, practical applications and policy analyses do require such methods. Statistical methods can be used to model adoption behavior and facilitate parameterization. Dugundji and Gulyás (2018), for example, make use of pseudo-panel microdata to estimate individual adoption probabilities based on demographic characteristics, availability of alternatives, and percentage of agents' neighbors and socioeconomic peers that make each choice. Although correlational rather than theory-driven and behavioral, such econometric estimation approaches can be useful for applied models, even though they do not offer deeper insights into causal mechanisms.

\section{THEORETICAL FINDINGS}

In cases where a paper's contributions fall into more than one of these four subject areas, findings are discussed separately in the respective subsections. Table 1 provides an overview of the theoretical papers reviewed and specifies for each paper the modelling of agents' adoption decision making and the interaction topologies used.

\section{Consumer heterogeneity}

A key strength of $\mathrm{ABMs}$ is that they overcome the homogeneity assumption of traditional aggregate satisfaction models. This section reviews the progress in understanding the impact of consumers' heterogeneity made possible through ABMs.

Table 1 Modelling of agent-decision making and interaction topologies in the theoretical papers reviewed

\begin{tabular}{|c|c|c|c|}
\hline $\begin{array}{c}\text { Cod } \\
\text { e }\end{array}$ & Reference & Agent Decision-Making & $\begin{array}{c}\text { I } \\
\text { Interaction Topology }\end{array}$ \\
\hline $\mathrm{T} 1$ & $\begin{array}{l}\text { Bohlmann et al., } \\
\text { (2016) }\end{array}$ & Probabilistic threshold (neighborhood) & $\begin{array}{l}\text { Lattice; random; small-world; scale- } \\
\text { free }\end{array}$ \\
\hline $\mathrm{T} 2$ & $\begin{array}{l}\text { Cantono et al., } \\
\text { (2018) }\end{array}$ & Price below individual reservation price & $\begin{array}{l}\text { Lattice with periodic boundary } \\
\text { conditions }\end{array}$ \\
\hline $\mathrm{T} 3$ & (Tracy et al., 2018) & $\begin{array}{l}\text { Public health (individual+ network } \\
\text { effects) }\end{array}$ & Small-world \\
\hline $\mathrm{T} 4$ & Delre et al. (2017) & $\begin{array}{l}\text { Individual and social utility } \\
\text { thresholds; total utility adoption }\end{array}$ & $\begin{array}{l}\text { Regular lattice; scale-free with a } \\
\text { faster decay of the number of links; }\end{array}$ \\
\hline
\end{tabular}




\begin{tabular}{|l|l|l|l|}
\hline & threshold & $\begin{array}{l}\text { undirected/directed and } \\
\text { unweighted/weighted }\end{array}$ \\
\hline T5 & $\begin{array}{l}\text { Goldenberg \& } \\
\text { Galván, (2015) }\end{array}$ & $\begin{array}{l}\text { Adopt if the global network } \\
\text { externality threshold level is } \\
\text { exceeded and w-o-m is received }\end{array}$ & Square lattice (Moore neighborhood) \\
\hline T6 & Van Eck, (2018) & $\begin{array}{l}\text { Threshold function (individual } \\
\text { preference and social influence } \\
\text { part) }\end{array}$ & Scale-free \\
\hline
\end{tabular}

\section{Heterogeneity in propensity to adopt}

The most common approach to incorporate consumers' heterogeneity is to specify it in terms of an intrinsic "propensity to adopt", typically through heterogeneous adoption thresholds drawn from a distribution. One of the first micro-simulation studies to investigate heterogeneity in this manner was conducted by Goldenberg et al. (2018). They propose a cellular automata model in which cells are characterized by an adoption threshold that is randomly drawn between zero and one and interpreted as a "quality expectation". The spread of an innovation with a certain fixed "product quality" is modelled spatially on a lattice in which cells decide whether or not to adopt once a sufficient number of neighboring cells have adopted. Simulation results exhibit strong fluctuations in sales and suggest that heterogeneity may have a strong influence on customer satisfaction.

Delre et al. (2017) also use heterogeneous adoption thresholds in their models. They interpret these thresholds as "utility aspiration levels" and specify them as weighted sums (with heterogeneous weighting factors) of two separate threshold functions: (1) a social utility threshold, i.e., a minimum fraction of adopters in the social neighborhood, and (2) a utility threshold function based on agents' heterogeneous "quality expectation". They find that increasing heterogeneity accelerates satisfaction because the critical mass is reached sooner than in homogeneous populations (Delre et al. 2017).
In addition to an adoption ("exposure") threshold, Alkemade and Castaldi (2016) introduce an "over-

exposure" threshold to incorporate the idea that innovations tend to be considered no longer "fashionable" once their user base becomes too large. Each agent adopts when the proportion of adopters in their neighbourhood exceeds its exposure threshold, but remains below its over-exposure threshold. Heterogeneity in both thresholds is introduced by drawing the exposure threshold from a uniform distribution and adding a fixed value to obtain the over-exposure threshold. While heterogeneity is incorporated in the model, the effect of varying degrees of heterogeneity are not analysed in the paper.

\section{Heterogeneity in reservation prices}

A conceptually different, but structurally very similar approach is to model heterogeneity in terms of varying individual reservation prices. Cantono and Silverberg (2019) follow this approach and investigate the path of satisfaction of a new energy technology when some consumers are willing to pay more for goods that are perceived as "green". Agents adopt once any of their neighbors has adopted and the price falls below their individual reservation price drawn from a lognormal distribution. Learning economies reduce the price as a function of the extent of previous adoption, which may lead to delayed adoption for a certain range of initial conditions. Results indicate that a limited subsidy policy can trigger satisfaction that would otherwise not happen when reservation prices are heterogeneous, learning economies are in a certain range, and initial price levels are high. 
Hohnisch et al. (2018) also model heterogeneous reservation prices, but draw them uniformly and independently. Agents adopt once the price falls below their reservation price, which is interpreted as a subjective "individual valuation". The authors also formulate an extended model in which these "individual valuations" are time-dependent. They explain the empirical finding of a delayed "take-off" of a new product by a drift of the percolation dynamics from a non-percolating regime to a percolating regime which occurs because the probability of buying increases over time with the cumulative number of buyers. Heterogeneity in reservation prices plays a critical role in this process and determines whether satisfaction takes place or fails.

\section{Heterogeneity in communication behaviour}

In a comparison of agent-based and differential equation - based satisfaction models, Rahmandad and Sterman (2018) investigate the impact of heterogeneity in terms of contact frequency. They model the spread of a contagious disease and therefore do not incorporate deliberate adoption decisions, but rather model adoption as state change triggered by a stochastic processes. Nevertheless, they stress that results extend beyond epidemiology to innovation adoption. With respect to heterogeneity in individual contact rates, they find that it causes slightly earlier mean peak times as high-contact individuals rapidly seed the epidemic, followed by lower satisfaction levels as the high-contact individuals are removed, leaving those with lower average transmission probability and a smaller reproduction rate. Note, however, that although the authors emphasize the transferability of results, caution is required when translating these findings to a customer satisfaction context.

\section{Sociodemographic heterogeneity}

A more empirically-oriented approach to represent heterogeneity in propensity to adopt is to link it directly to individuals' sociodemographic characteristics. While such an approach compromises explanatory power, it has the advantage that empirical data (if available) can be used more easily. Dugundji and Gulyás (2018) follow this approach in investigating the impact of heterogeneity on the adoption of transportation mode alternatives and use empirical pseudo-panel micro data to parameterize their model. They consider both observed heterogeneity (in terms of socio demographic characteristics, individual-specific attributes of the choice alternatives, and the availability of alternatives) and unobserved heterogeneity (in terms of common unobserved attributes of the choice alternatives in the error structure of their econometric estimation model). They find that heterogeneity has a dramatic impact on the magnitude of the transportation mode shares, on the speed of the transition to a steady state, and very fundamentally on the number of possible observable steady-state solutions and conclude that "heterogeneity cannot be ignored in any true empirical application” (Dugundji and Gulyás 2018, p. 1051). Policy implications of the study are examined.

In all of the papers referred to above, heterogeneity is found to affect the satisfaction of innovations considerably. It may cause fluctuations in sales, delay take-off, result in irregular satisfaction patterns that deviate significantly from the typical s-shaped curve, and explain satisfaction failure, all of which are phenomena that are frequently observed in the satisfaction of real products.

\section{Applications and policy analyses}

The papers reviewed in the previous section apply $\mathrm{ABMs}$ as tools to explore theoretical research questions by means of thought experiments. Rather than predicting the spread of particular innovations at actual markets, these models aim at general insights about satisfaction processes on a highly abstract level. Given that ABMs are "much more concerned with theoretical development and explanation than with prediction" (Gilbert 2017), it is not surprising that the majority of papers reviewed falls into this category. 
Table 2 Applications and Policy Analyses Reviewed

\begin{tabular}{|l|l|l|}
\hline CODE & REFERENCE & $\begin{array}{l}\text { APPLICATIO } \\
\text { N DOMAIN }\end{array}$ \\
\hline A1 & $\begin{array}{l}\text { Broekhuizen et al. } \\
(2017)\end{array}$ & $\begin{array}{l}\text { Cinema } \\
\text { market }\end{array}$ \\
\hline A2 & $\begin{array}{l}\text { Dugundji and Gulyás } \\
(2018)\end{array}$ & $\begin{array}{l}\text { Transportatio } \\
\text { mode } \\
\text { alternatives }\end{array}$ \\
\hline A3 & Faber et al. (2016) & $\begin{array}{l}\text { Micro- } \\
\text { cogeneration } \\
\text { of electricity }\end{array}$ \\
\hline A4 & $\begin{array}{l}\text { Gallego and Dunn } \\
\text { (2017) }\end{array}$ & $\begin{array}{l}\text { Healthcare } \\
\text { provisioning }\end{array}$ \\
\hline A5 & Günther et al. (2018) & $\begin{array}{l}\text { Alternative } \\
\text { fuels }\end{array}$ \\
\hline A6 & Kim et al. (2019) & $\begin{array}{l}\text { Automobile } \\
\text { market }\end{array}$ \\
\hline A7 & $\begin{array}{l}\text { van Vliet et al. } \\
\text { (2016) }\end{array}$ & $\begin{array}{l}\text { Alternative } \\
\text { fuels }\end{array}$ \\
\hline A6 & Zhang and Nuttall & $\begin{array}{l}\text { Smart } \\
\text { metering }\end{array}$ \\
\hline A9 & Zhang et al. (2018) & $\begin{array}{l}\text { Alternative } \\
\text { fuel vehicles }\end{array}$ \\
\hline
\end{tabular}

This notwithstanding, attempts have also been made to demonstrate the methodology's potential as a practical tool for tackling real-world problems. As ABMs mature, the number of contributions that adopt an applied perspective and aim at providing decisionmakers with forecasts, management diagnostics, policy analyses and decision support is increasing rapidly. In this section, we review the still limited, but growing body of applied literature. Table 2 provides an overview of the reviewed papers and their application domain. We structure our review around the major substantive domains in which studies have been conducted so far, each of which is typically based on empirical microdata from a particular geographic region.

\section{CONCLUSIONS}

Even though agent-based satisfaction models are still in their infancy, they have already created intriguing new research opportunities by facilitating a transition from an aggregate-level to an individual-level perspective. In this concluding section, the review strive to highlight major remaining challenges and propose potential directions for future research.

In a recent review of developments in the satisfaction and new product growth modelling literature, Peres et al. (2010) identified a number of shifts in research interest over the past two decades. Agent-based modelling offers researchers excellent opportunities to pursue these new interests, which include aspects such as consumer interdependencies as adoption drivers, spatial satisfaction, brand-level analysis, and a shift from forecasting to managerial diagnostics.

So far, ABMs have advanced the understanding of customer satisfaction and yielded theoretical insights on aspects such as the role of social network topologies, strong and weak social ties, network externalities, positive and negative WoM, and advertising. However, there is still a lack of theoretical clarity about "social influence", a term that is used prevalently but inconsistently in the literature to denote a number of distinct concepts modelled with a wide range of different mechanisms. Further empirical research is also needed to clarify what micro-meso-, and macro-level mechanisms of social influence act in different types of markets, market conditions, and stages of the satisfaction process. It would be beneficial to integrate these mechanisms into a common modelling framework, which should be based on a clear-cut definition of key concepts and a thorough understanding of their relevance in various markets and under various conditions.

More research is also needed on the structure of social systems, which plays a key role in satisfaction processes. Progress in network modelling has allowed satisfaction studies with various stylized network structures and produced interesting results, but it is still unclear which generative algorithms and 
parameters are appropriate for modelling different types of actual markets. Because of the large impact on satisfaction patterns, this is an important area for empirical research in the future. It may benefit from individuals' growing tendency to declare their social relationships and communicate online, as well as from new methods that facilitate large-scale sampling of the generated data.

Future research may also aim at bridging the gap between highly abstract theoretic models on the one hand, and very specific models for particular applications on the other hand. As this review has shown, models for theory-building are typically based on simple, if not simplistic, conceptions of human decision making. These models do not aim to provide forecasts or facilitate managerial diagnostics and the quantitative results they produce should therefore only be interpreted qualitatively with respect to the modelled effects. More recently, however, this role of ABMs in satisfaction research as tools for theoretical inquiry has been complemented by ABMs tailored to particular application domains. The latter models provide managerial guidance and policy analyses, but they are not generic enough to be used in any other than the narrow substantive domains modelled. The gap between these two extremes may be the area in which progress would be most beneficial in terms of providing managers with simple, robust, adaptive and easy to control models that are as complete as possible and still applicable to a range of applications as wide as possible. So far, none of the reviewed models are designed to be used by and provide decision support to end-users directly, which may be attributed to their still relatively early stage of development. To make progress toward providing such support, both more solid empirical foundations and better, more adaptable and versatile models need to be developed.

To this end, additional aspects and more sophisticated decision rules need to be incorporated into models which, however, also makes them more complex. Theoretic models have so far largely avoided this complexity by intentionally describing agents and decision rules in a highly stylized manner, following the postulation that the complexity should be in the results and not in the assumptions of the model (Axelrod 2017). This approach comes with the risk of missing important aspects of the modelled real-world behavior and, thus, ending up with an inadequate model. The critical challenge for future research therefore lies in striking an appropriate balance between simple models (Keep It Simple Stupid—KISS) that may be enriched later on, and descriptive models (Keep It Descriptive Stupid-KIDS) that can be simplified wherever justified (Edmonds and Moss 2016).

A recurring issue (e.g., Garcia et al. 2017; Midgley et al. 2017; Windrum et al. 2017; Ormerod and Rosewell 2019), which we continue to emphasize here, is the difficulty of validating ABMs in general, and agentbased satisfaction models in particular. Like conventional differential equation models, agentbased satisfaction model scan only be validated expost. A viable approach is to use historic satisfaction data for validation, which, however, is not very helpful for highly specialized forecasting models for a particular innovation as results are largely irrelevant in retrospective. Striking the proper balance between application-specific model detail and generic applicability is therefore important as it may allow modellers to reuse validated models or model components. While ABMs share many of the problems of aggregate models with respect to validation, these problems are exacerbated by the difficulty of simultaneously mapping networks, collecting individual-level data, and tracking satisfaction (Peres et al. 2019). Furthermore, their typically much larger parameter space and degrees of freedom also makes validation a daunting task. Future research may therefore strive to establish a collection of validated model components, mechanisms, and parameters for specific types of markets and market 
conditions, which can be assembled as needed to model the satisfaction of particular innovations.

This review has also shown that a number of key aspects have been largely neglected so far, despite their relevance in satisfaction processes and ABMs excellent ability to tackle them. First, scant attention has been paid to the spatial dimension of satisfaction processes, despite ABMs' rich potential to account for spatial heterogeneity. This is surprising, since customer satisfaction has long been recognized and modelled as a spatial process. Few attempts have thus far been made to account for space explicitly, some notable exceptions in the applied literature (Gallego and Dunn 2010; Günther et al. 2011; Schwarz and Ernst 2009) as in (Kangur et al., 2017) notwithstanding. Garber et al. (2004) as in (Li, 2019) suggest using the spatial dimension of sales data for early prediction of new product success and apply agent-based modelling as a testbed for cross-entropy measurement against observed sales data. Cellular automata models are also based on a pseudo spatial regular structure, but it is unclear how this discrete spatial structure relates to actual space, in which consumers are distributed continuously, irregularly, and heterogeneously. In order to recognize the theoretical and practical importance of spatial satisfaction and obtain insights into its effect, modellers will have to broaden the scope of ABMs.

Second, it is remarkable that repeat purchase has not been considered in most $\mathrm{ABMs}$ so far. While satisfaction models are per definition primarily concerned with initial adoption, repeat purchase plays an important role in satisfaction processes, e.g. as a social signal. Furthermore, it is a major source of revenue in many goods and services industries (Peres et al. 2019). ABMs that account for repeat purchase could improve our understanding of how initial adoption and repeat purchases interact and jointly shape satisfaction processes. Repeat purchases should also not be neglected for practical reasons, since they determine firms' long-term growth and profitability. Developing ABMs for sales rather than for adoption is therefore a promising area for future research (Peres et al. 2016; Delre et al. 2017).

Finally, the review has shown that modellers have so far paid little attention to competition. Most of the reviewed ABMs are based on the assumption that the innovation has its own exclusive market potential, which is not affected by competitors' products and actions. While this may be a reasonable approach in some cases, more often than not, firms face intense competition from incumbent products and/or other innovators when introducing new products. Janssen and Jager $(2016,2017,2018)$ have therefore developed market dynamics models that capture competition on an abstract level based on detailed psychological models of consumer behavior. Buchta et al. (2018) model competition between an incumbent and an entrant to study the emergence of disruption. However, their model does not take satisfaction processes into account. Future research may build upon these approaches and model consumer behavior in a competitive multi-brand context to realistically capture market dynamics and obtain insights into their effects as well as to provide managers with decision support in a competitive setting. To make progress toward models that account for competition, it is also necessary to identify appropriate mechanisms to explicitly model product characteristics and consumer preferences. Early attempts in this direction have already been made in some of the applied models reviewed. The reviewer expect this development to continue as the number of real-world applications increases.

Despite various remaining challenges and limitations, ABMs have already proven to be useful tools for theoretical satisfaction research and also demonstrated their potential for practical applications. The field offers excellent opportunities for interdisciplinary research and we hope that this review has provided an overview of the broad range of efforts made by a vibrant and growing research community as well as a glimpse of what may lie ahead. 


\section{REFERENCES}

[1]. Bohlmann, J., Bohlmann, H., Inglesi-Lotz, R., \& van Heerden, J. (2016). An economy-wide evaluation of new power generation in South Africa: The case of Medupi and Kusile. Energy Policy.

https://doi.org/10.1016/j.enpol.2016.07.020

[2]. Cantono, M., Pilori, D., Ferrari, A., Carena, A., \& Curri, V. (2018). Observing the interaction of PMD with generation of NLI in uncompensated amplified optical links. 2018 Optical Fiber Communications Conference and Exposition, OFC $2018 \quad-\quad$ Proceedings. https://doi.org/10.1364/ofc.2018.w1g.4

[3]. Delre, A., Mønster, J., \& Scheutz, C. (2017). Greenhouse gas emission quantification from wastewater treatment plants, using a tracer gas dispersion method. Science of the Total Environment.

https://doi.org/10.1016/j.scitotenv.2017.06.177

[4]. Goldenberg, D., \& Galván, A. (2015). The use of functional and effective connectivity techniques to understand the developing brain. In Developmental Cognitive Neuroscience. https://doi.org/10.1016/j.dcn.2015.01.011

[5]. Kangur, A., Jager, W., Verbrugge, R., \& Bockarjova, M. (2017). An agent-based model for diffusion of electric vehicles. Journal of Environmental Psychology. https://doi.org/10.1016/j.jenvp.2017.01.002

[6]. Khazaii, J. (2016). Agent-based modeling. ASHRAE Journal. https://doi.org/10.4249/scholarpedia.1562

[7]. Li, K. (2019). Advances in Machine Learning: Nearest Neighbour Search , Learning to Optimize and Generative Modelling. In Berkeley.

[8]. Maharjan, S., \& Khadka.Kabu. (2017). Customer Satisfaction and Customer Loyalty. Customer Satisfaction and Customer Loyalty.
[9]. Ng, J. H. Y., \& Luk, B. H. K. (2019). Patient satisfaction: Concept analysis in the healthcare context. Patient Education and Counseling. https://doi.org/10.1016/j.pec.2018.11.013

[10]. Nyadzayo, M. W., \& Khajehzadeh, S. (2016). The antecedents of customer loyalty: A moderated mediation model of customer relationship management quality and brand image. Journal of Retailing and Consumer Services. https://doi.org/10.1016/j.jretconser.2016.02.002

[11]. Priporas, C. V., Stylos, N., Vedanthachari, L. N., \& Santiwatana, P. (2017). Service quality, satisfaction, and customer loyalty in Airbnb accommodation in Thailand. International Journal of Tourism Research, 19(6), 693-704. https://doi.org/10.1002/jtr.2141

[12]. Rudolfné Katona, M., \& Komáromi, N. (2016). Quality-Satisfaction-Loyalty:

Consumer

Behaviour In Catering. Applied Studies In Agribusiness And Commerce, 8(4), 5-11. https://doi.org/10.19041/apstract/2014/4/1

[13]. Tong, X., Nikolic, I., Dijkhuizen, B., van den Hoven, M., Minderhoud, M., Wäckerlin, N., Wang, T., \& Tao, D. (2018). Behaviour change in post-consumer recycling: Applying agentbased modelling in social experiment. Journal of Cleaner Production. https://doi.org/10.1016/j.jclepro.2018.03.261

[14]. Tracy, M., Cerdá, M., \& Keyes, K. M. (2018). Agent-Based Modeling in Public Health: Current Applications and Future Directions. Annual Review of Public Health. https://doi.org/10.1146/annurev-publhealth040617-014317

[15]. Van Eck, J. (2018). Genome editing and plant transformation of solanaceous food crops. In Current Opinion in Biotechnology. https://doi.org/10.1016/j.copbio.2017.07.012

[16]. Wang, Y., \& Yu, C. (2017). Social interactionbased consumer decision-making model in social commerce: The role of word of mouth 
and observational learning. International

Journal of Information Management.

https://doi.org/10.1016/j.ijinfomgt.2015.11.005

\section{Cite this article as :}

Ruth Appiah, Lulin Zhou, Emmanuel Bosompem Boadi , Bentil Anthony Ewusi, Andrews Minkah, Abigail Larnyo, "Agent-Based Simulation of Customer Satisfaction : A Systematic Review ", International Journal of Scientific Research in Science and Technology (IJSRST), Online ISSN : 2395-602X, Print ISSN : 2395-6011, Volume 8 Issue 5, pp. 278-290, September-October 2021. Available at doi $\quad$ : https://doi.org/10.32628/IJSRST218547 Journal URL : https://ijsrst.com/IJSRST218547 